\title{
A comparison of health-related quality of life between children with versus without special health care needs, and children requiring versus not requiring psychiatric services
}

\author{
Meichun Mohler-Kuo • Michelle Dey
}

Accepted: 27 November 2011 / Published online: 14 December 2011

(C) Springer Science+Business Media B.V. 2011

\begin{abstract}
Purpose The purpose of the present study was to compare health-related quality of life (HRQoL) between children with special health care needs (CSHCN) and those without. In particular, $\mathrm{CSHCN}$ who require psychiatric services and those who do not were compared.

Methods A representative community sample of 3,325 children (10-14 years old) was recruited from the Canton of Zurich. Via either computer-assisted telephone interviews or a written questionnaire, special health care needs were assessed using the five-item parent-reported CSHCN Screener. Subsequent to screening, a written questionnaire was sent to a sub-sample of 974 children to acquire more detailed information, both from the children and their parents, about their health and health care utilization, and to assess HRQoL (KIDSCREEN-27) and emotional and behavioral problems (SDQ). A total of 626 children responded to the questionnaire. Multiple linear regression models were used to examine the association between HRQoL and subject group, controlling for other covariates. Results Among 2,586 children whose parents participated in the screening stage, roughly $18 \%$ were identified as CSHCN, with $6.2 \%$ requiring psychiatric services. The subsequent survey revealed that those CSHCN who required psychiatric services had the lowest HRQoL scores and highest SDQ scores. CSHCN who utilized psychiatric services were particularly prone to low HRQoL, especially among lower income families.
\end{abstract}

M. Mohler-Kuo $(\square) \cdot$ M. Dey

Institute of Social and Preventive Medicine, University of Zurich, Hirschengraben 84, 8001 Zürich, Switzerland

e-mail: meichun.mohler-kuo@uzh.ch
Conclusions The influence of noticeable emotional and behavioral problems on HRQoL should be afforded more attention, both in clinical practice and empirical studies involving children with special health care needs.

Keywords Special health care needs - Psychiatric services - Mental health problems - Health-related quality of life · Children

$\begin{array}{ll}\text { Abbreviations } \\ \text { CSHCN } & \text { Children with special health care needs } \\ \text { HRQoL } & \text { Health-related quality of life } \\ \text { CATI } & \text { Computer-assisted telephone interviews } \\ \text { SDQ } & \text { Strength and Difficulties Questionnaire } \\ \text { FAS } & \text { Family Affluence Scale } \\ \text { SES } & \text { Socioeconomic status }\end{array}$

\section{Introduction}

The World Health Organization (WHO) defines "health" as a state of complete physical, mental, and social wellbeing, and not merely the absence of disease or infirmity [1]. Hence, health is not only considered on the basis of somatic indicators but also on how a person feels, psychologically and physically, and how he/she interacts with other persons and functions in everyday life. This perceived health is known as "health-related quality of life" (HRQoL). HRQoL includes a multi-factorial construct to describe individuals' perception of their physical, psychological, and social functioning. It is especially important among children with a chronic condition. Chronic health conditions among children and adolescents are stressful 
adversities that can have significant psychosocial and developmental impacts upon the children or adolescents themselves, and upon their families, as well. In addition to limitations related to their condition, they are at higher risk for various negative outcomes, like mental and behavioral health problems, increased school absences, and unmet health care needs [2-8].

A better understanding of quality of life from children's perspective could help in planning and assessing the effectiveness of health care or other interventions. Despite the lack of any universally accepted definition of HRQoL, in the context of chronic conditions affecting children, the following definition by Taylor et al. [9] seems to be appropriate, because it not only describes the subjective and multidimensional nature of HRQoL but also its dynamics. Moreover, it considers the influences of the health condition and the developmental stage of the child:

HRQoL in young people with chronic illness is subjective, multidimensional and dynamic. It is unique to each individual young person and includes aspects of physical, psychological and social function. It depends upon not only the stage of development but also the illness trajectory.

In recent years, there has been growing interest in the area of HRQoL among children, because of the so-called "new morbidity" (described as a shift to place importance on the chronic disease management and the social and behavioral issues as the consequences of chronic conditions [10]). This new morbidity and the treatments for particular conditions not only require the collection of objective criteria (e.g., mortality, morbidity, and symptoms), but a broader subjective evaluation (e.g., a HRQoL assessment) in order to obtain a comprehensive overview of the impact of the health condition and the effects of a given treatment [10-12].

Although growing interest in HRQoL is apparent, several research gaps remain unaddressed [12-14]: First, only about $13-15 \%$ of the research that has been published to date has targeted children. Second, there is a lack of published studies examining the HRQoL of children with mental health problems; most studies have focused exclusively on the examination of HRQoL among children with physical illness. The few available studies suggest that mental disorders in children negatively affect HRQoL (e.g., [15-20]). However, significant limitations are apparent in this research field [19, 21]. First, most studies have involved clinically based samples, and it is likely that a bias toward poorer HRQoL is present among young people recruited from psychiatric clinics. Second, most studies have used parents as proxy respondents, even though the subjective views of the child are very important (particularly if a child suffers from an internalized mental condition) and children's perspectives of their HRQoL generally are as valid as the appraisals of their parents [22].

The present study aims to compare HRQoL between children aged 10-14 with and without special health care needs $(\mathrm{CSHCN})$, using a representative sample drawn from municipalities. There has been a lack of quality data focusing specifically on this age group, as most health surveys have targeted respondents 15 years old or older. Furthermore, selecting this age group, as opposed to younger children, allows us to obtain the assessment of HRQoL directly from the children themselves. In particular, we will compare HRQoL between CSHCN who require psychiatric services and those who do not.

In 1998, the US Maternal and Child Health Bureau published a new definition of CSHCN: "Children who have special health needs are those who have or are at increased risk for a chronic physical, developmental, behavioral or emotional condition and who also require health and related services of a type or amount beyond that required by children generally" [23]. According to this definition, children are identified based upon experiencing one or more current functional limitations or service use needs that are the direct result of an ongoing physical, emotional, behavioral, developmental, or other health condition. Such a non-categorical approach is now being widely accepted in epidemiological research, health services research, and welfare/health programs for children, because it considers that different chronic conditions often lead to similar consequences (e.g., greater healthcare utilization, increased burden to parents, lower HRQoL); hence, an aggregation of different chronic conditions is appropriate [24, 25]. Furthermore, the non-categorical approach makes it possible for even very rare diseases to be considered in epidemiological studies.

A recent study conducted in Switzerland [16] indicated that CSHCN have a lower level of HRQoL than healthy controls. Comparable results have been found in international investigations [26]. However, these studies did not compare the HRQoL of CSHCN who had required versus not required psychiatric services. A study conducted in the United States identified reduced HRQoL in CSHCN with emotional, behavioral, or developmental problems compared to CSHCN without such conditions [17]. However, in this study, each child's HRQoL was rated by the parents.

In summary, to address above-mentioned research gaps, the present study aims to compare HRQoL of CSHCN with healthy controls. In particular, we will further compare the HRQoL of CSHCN who require psychiatric services and CSHCN who do not. We expect that the results of the present study will contribute to a better understanding of HRQoL among CSHCN requiring psychiatric services from the child's point of view, a perspective that has not been examined adequately to date. 


\section{Methods}

\section{Study design}

This epidemiological study was conducted in 2007 in the Canton of Zurich. The study had two phases. Phase I consisted of screening to identify CSHCN. Phase II entailed a subsequent survey of a subsample of those who had been screened to obtain more detailed information on health indicators of CSHCN. The present paper will primarily report the results of Phase II.

\section{Sampling}

Our initial intention was to screen roughly 3,500 children, ages $10-14$, so as to collect a sample of at least 200 CSHCN to participate in Phase II. Two-stage sampling was used to select the Phase I sample. In the first sampling stage, using the Bernese sampling plan [27], we selected 35 out of a total of 171 municipalities in the Canton of Zurich to participate in the study. Details of the study design and sampling strategy, including the representativeness of the sample, have been published elsewhere [28]. As the second stage, we asked the selected municipalities to draw a random sample of children who met our age criteria (year of birth: 1993-1997) from their registry of residents and provide us with information about the child (last name, first name, birthdate, gender, address, and nationality) and parents (last and first name of the mother and/or father, telephone number). Thirty-three of the 35 selected municipalities supplied the requested information (collaboration rate $=94 \%$ ), generating a Phase I sample of 3,341 randomly selected children. We subsequently excluded 16 children from the sample because they were abroad or deceased or had moved out of the selected municipalities. Consequently, a final sample of 3,325 children was available for screening. Figure $1 \mathrm{a}$ and $\mathrm{b}$ depicts the sampling procedures for the Phase I screening and Phase II subsample survey, respectively.

Procedures

\section{Phase I: Screening for special health care needs}

We used the five-item parent-reported CSHCN Screener to identify children with chronic conditions [23] and computer-assisted telephone interviews (CATI) for data collection during the screening procedure. However, if the telephone numbers of families were not available, the CSHCN Screener was mailed to the family, accompanied by a return envelope. As illustrated in Fig. 1a, 3,011 telephone numbers were identified, among which 2,864 were a

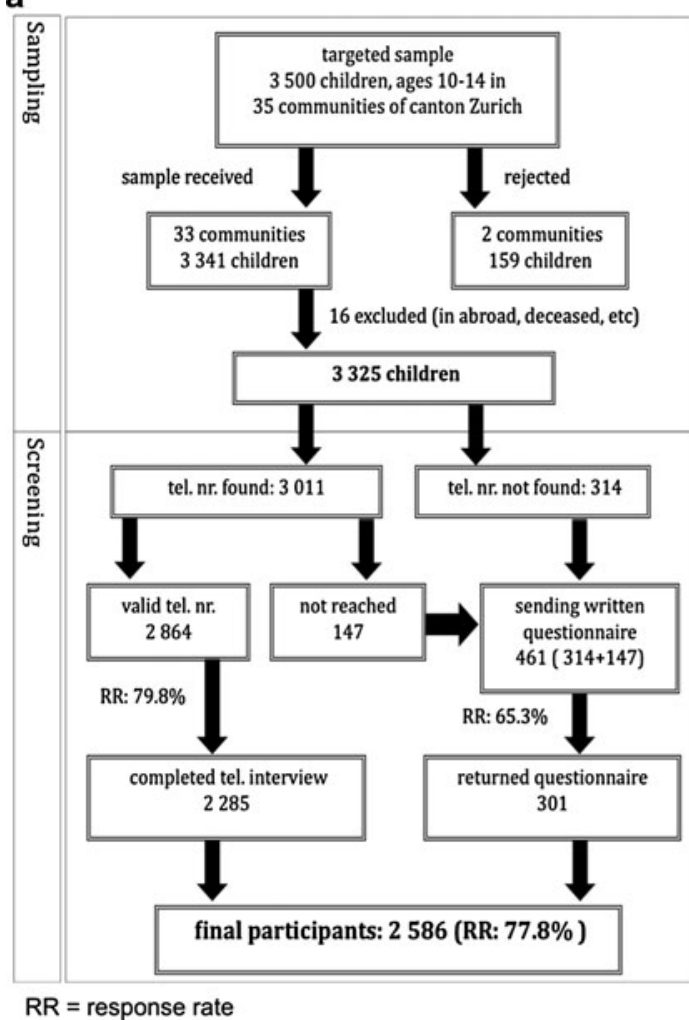

b

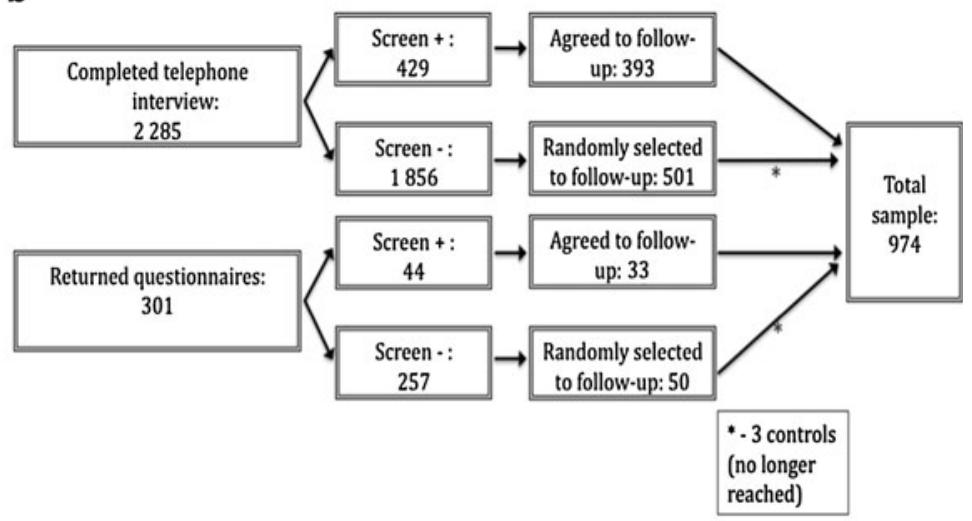

Fig. 1 a Sampling procedures for Phase I. b Sampling procedures for Phase II 
valid. A total of 2,285 of the parents completed the telephone interviews (response rate $=79.8 \%$ ). Out of 461 families (314 families whose telephone numbers could not be found and an additional 147 families who were not reached by telephone), 301 families returned the questionnaire (response rate $=65.3 \%$ ). Altogether, 2,586 families participated in the first phase of the study (response rate $=77.8 \%$ ). As reported in a previous publication [28], the demographic distribution of the 2,586 respondents was quite similar to the total population of children 10-14 in Canton Zurich, in terms of gender and age. A slightly lower percentage of non-Swiss respondents (18\%) participated in the screening phase, relative to the total population of children 10-14 in Canton Zurich (22\%).

\section{Phase II: Post-screen sub-sample survey}

Participants: Among 2,586 children who were screened, 473 qualified as CSHCN (161 required psychiatric services; 312 did not) and 2,113 were classified as children without special health care needs. We invited all of the CSHCN to participate in the sub-sample survey, so as to permit an even better understanding of CSHCN. As shown in Fig. 1b, Phase II questionnaires were sent to 426 CSHCN whose parents, at the time of screening, had agreed to participate in the sub-sample study. An additional 551 children without special health care needs were chosen randomly to participate in the Phase II survey as a control group. We excluded 3 families from the control group because they no longer could be reached. Therefore, follow-up questionnaires were sent to a total of 974 families (426 CSHCN; 548 controls). Participation was voluntary. Parents and children consented to participate in the subsample survey by sending the questionnaires back. A total of 694 families returned a completed parent and/or child questionnaire (response rate $=71.2 \%$; 687 parent questionnaires; 626 child questionnaires). Among them, 291 families had a CSHCN and 403 were considered controls (response rates $=68.3$ and $73.5 \%$, respectively). Due to regulations imposed by the Data Protection Committee and the Municipalities of Canton Zurich, the only information available to us on non-respondents was sex, age, nationality (available from only $85 \%$ of the municipalities), and clinical status (case vs. non-case), which was obtained from the telephone interview during the screening procedure. Our analysis revealed that participation in the subsample survey was not influenced by child age $\left(\chi^{2}(4\right.$, $N=974)=3.58, P=0.47)$, gender $\left(\chi^{2}(1, N=974)=\right.$ $0.86, \quad P=0.36)$, nationality $\left(\chi^{2}(1, \quad N=830)=3.56\right.$, $P=0.06)$, or clinical status $\left(\chi^{2}(1, N=974)=2.64\right.$, $P=0.10)$ according to responses obtained from the parent survey. Although parents of non-Swiss nationality were less likely to respond to the survey than those of Swiss nationality ( $62 \%$ vs. $70 \%$, respectively), this difference was not statistically significant, as shown previously $(P=0.06)$. Similar results were found for the children's questionnaire, except that the participation rate of children was significantly lower among cases versus controls, because many children were not able to fill out the questionnaire due to their health condition $\left(\chi^{2}(1, N=974)=\right.$ 7.86, $P=0.005)$.

\section{Measurements}

Screening Instrument: "Children with Special Health Care Needs (CSHCN) Screener" is a five-item, parent-based survey instrument to screen children and adolescents with chronic health conditions [29]. The screening instrument was developed specifically to reflect the US Maternal and Child Health Bureau's definition of children with special health care needs, and it has been widely used and validated in the US National Survey of Children with Special Health Care Needs. It was also recently validated in a study in which seven European countries participated [30]. According to this definition, children are identified on the basis of experiencing one or more current functional limitations or service use needs that are the direct result of an ongoing physical, emotional, behavioral, developmental, or other health condition, rather than a specific diagnosis. A child is classified as having special health care needs if he/ she presently experiences at least one of the following health consequences: (1) the need for or use of prescribed medicine; (2) the need for or use of medical care, mental health, or educational services; (3) the presence of functional limitations; (4) the need for or use of special therapies; and (5) the need for or use of treatment or counseling for emotional, developmental, or behavioral problems. These health consequences must be due to a medical, behavioral, or other health condition that has lasted or is expected to last for at least 12 months. We further divided those children identified as CSHCN into two subgroups: $\mathrm{CSHCN}$ requiring and $\mathrm{CSHCN}$ not requiring psychiatric services. A CSHCN was considered to be in the subgroup requiring psychiatric services if the fifth item on the Screener was positive, regardless of answers to the other questions [17]. Therefore, this subgroup included CSHCN who either only had psychological problems or who had both psychological problems and other chronic physical conditions. All other CSHCN would be considered to belong in the subgroup not needing psychiatric services (i.e., the fifth item screened negative).

Questionnaires for the Phase II sub-sample survey: Separate Phase II questionnaires were filled out by the parent (or parents) and the child. The questionnaire for parents included items on social demographics, as well as the child's functional status, living situation, health care 
utilization and satisfaction, health insurance, and welfare support. The child's questionnaire included the following measures:

- KIDSCREEN-27 [26]: We used the KIDSCREEN-27 to measure HRQoL. The KIDSCREEN-27 has been validated internationally, including Switzerland [26]. It is comprised of 5 subscales, pertaining to "physical wellbeing" (5 items), "psychological well-being" (7 items), "autonomy and parent relations" (7 items), "social support and peers," (4 items) and "school environment" (4 items). In the present study, children responded to each item on the KIDSCREEN-27 using a 5-point response scale, with eligible scores ranging from " 1 " (not at all/ never) to " 5 " (extremely/always). To calculate summation scores for the different scales, reverse scoring was used for 4 items. Because the 5 sub-scales differ in the number of items, the summation score for each subscale was standardized [26], thereby generating an overall standardized raw score with values ranging between 0 and 100 , with higher scores indicating a better self-reported HRQoL.

- Strength and Difficulties Questionnaire (SDQ) [31]: The SDQ is a behavioral screening questionnaire that has been validated in different countries and different samples (e.g., [32, 33]). It includes 25 items (positive and negative attributes) within the following 5 subscales: "hyperactivity," "emotional symptoms," "conduct problems," "peer problems," and "prosocial." Each item is scored using a 3-point response scale (for 20 of the 25 items: $0=$ not true, $1=$ somewhat true, $2=$ certainly true). A total difficulties score (ranging from 0 to 40) is calculated, whereby all but the "prosocial scale" score are combined. Based upon defined cut-off-scores, the scale summation scores and the total difficulties score can be subdivided into "normal," "borderline," and "abnormal."

- Family Affluence Scale (FAS) [34]: We used the FAS, which was completed by the children to measure their family's socioeconomic status (SES). The scale contains 4 questions: (1) Does your family own a car, van, or truck? (response categories: no $=0$; yes $=1$; yes, two or more $=2)$; (2) Do you have your own bedroom? (response categories: no $=0$; yes $=1$ ); (3) During the past 12 months, how many times did you travel away on holiday with your family? (response categories: not at all $=0$; once $=1$; twice $=2$; more than twice $=3$ ); and (4) How many computers does your family own? (response categories: none $=0$; one $=1$; two $=2$; more than two $=3$ ). The scale has been validated in multinational surveys in many countries [35]. A composite FAS score (range, 0-9) was calculated for each child according to his or her responses to these four items. The scale was further classified into a three-point ordinal scale, where a FAS score $=0,1$, or 2 indicates a low degree of affluence (poverty); a FAS score $=3,4$, or 5 indicates intermediate affluence; and a FAS score $=6,7,8$, or 9 indicates highly affluent.

Data analysis

All data analyses were conducted using SAS 9.2. Standardized mean scores were computed for each subscale of HRQoL, by sex and by subject group (CSHCN with psychiatric service use, CSHCN without psychiatric service use, and healthy controls). Mean SDQ scores also were computed for each subscale, again by sex and by subject group. Pearson correlation coefficients were calculated to assess the association between HRQoL and the SDQ. Multiple linear regression analyses were performed to compare the HRQoL of the three groups while adjusting for other correlates. The outcome variables were each of the 5 dimensions of HRQoL. In the model, the healthy control group was used as the baseline state, to compare their mean difference of HRQoL against the two CSHCN groups. Initially, the correlates that are clinically important to HRQoL were examined in each model; they are age, sex, nationality, family SES, and the education level of the father and mother. Because we observed different associations between clinical status and HRQoL by gender, an interaction term for "CSHCN requiring psychiatric services* girl" was included in the final regression model. In the final regression models (Table 4), only those correlates with a $P$ value $<0.1$ were included.

\section{Results}

\section{Study participants}

Table 1 shows the socio-demographic characteristics of all children and their parents who participated in the Phase II sub-sample survey. Among those 626 children who returned their questionnaires, 253 were $\mathrm{CSHCN}$, among whom 88 required psychiatric services $(70.5 \%$ boys; $29.6 \%$ girls) and 165 did not (64.9\% boys; $35.2 \%$ girls). There were 373 controls ( $46.4 \%$ boys; $53.6 \%$ girls). The mean age of the children was $12.8 \pm 1.4$ years. Roughly, three in four children were Swiss. One in five children among the CSHCN who required psychiatric services attended special classes; this percentage was lower in $\mathrm{CSHCN}$ not requiring psychiatric services and healthy controls (7.4 and 2.5\%, respectively). More families with $\mathrm{CSHCN}$ requiring psychiatric services were classified in the category of "low family affluence" (8.0\%) relative to CSHCN without 
Table 1 Socio-demographic characteristics of participating children and their parents

\begin{tabular}{|c|c|c|c|c|}
\hline & $\begin{array}{l}\text { Total } \\
(N=626)\end{array}$ & $\begin{array}{l}\text { CSHCN requiring } \\
\text { psychiatric services } \\
(N=88)\end{array}$ & $\begin{array}{l}\text { CSHCN without } \\
\text { psychiatric services } \\
(N=165)\end{array}$ & $\begin{array}{l}\text { Controls } \\
(N=373)\end{array}$ \\
\hline Age in years: mean (SD) & $12.8(1.4)$ & $12.9(1.4)$ & $12.8(1.4)$ & $12.7(1.4)$ \\
\hline \multicolumn{5}{|l|}{ Sex } \\
\hline Boy $(\%)$ & 54.6 & 70.5 & 64.9 & 46.4 \\
\hline Girl $(\%)$ & 45.4 & 29.6 & 35.2 & 53.6 \\
\hline \multicolumn{5}{|l|}{ Nationality } \\
\hline Swiss $(\%)$ & 71.9 & 77.0 & 80.0 & 67.2 \\
\hline Others $(\%)$ & 28.1 & 23.0 & 20.1 & 32.8 \\
\hline \multicolumn{5}{|l|}{ School attendance } \\
\hline Regular classes (\%) & 93.5 & 78.2 & 92.6 & 97.6 \\
\hline Small/special classes $(\%)$ & 6.5 & 21.8 & 7.4 & 2.5 \\
\hline \multicolumn{5}{|l|}{ Family SES (family affluence scale) } \\
\hline Low $(\%)$ & 4.8 & 8.0 & 3.7 & 4.6 \\
\hline Medium (\%) & 19.7 & 17.1 & 13.5 & 23.1 \\
\hline High $(\%)$ & 75.4 & 75.0 & 82.8 & 72.3 \\
\hline \multicolumn{5}{|l|}{ Education of father } \\
\hline Mandatory school (\%) & 6.0 & 9.4 & 4.4 & 5.9 \\
\hline Higher vocational/pre-college high school (\%) & 28.0 & 27.1 & 34.4 & 25.4 \\
\hline University/college (\%) & 27.8 & 24.7 & 26.9 & 29.0 \\
\hline \multicolumn{5}{|l|}{ Education of mother } \\
\hline Mandatory school (\%) & 7.2 & 9.3 & 5.0 & 7.7 \\
\hline Higher vocational/pre-college high school (\%) & 32.5 & 33.7 & 34.4 & 31.3 \\
\hline University/college (\%) & 13.9 & 16.3 & 11.9 & 14.3 \\
\hline
\end{tabular}

Values are expressed as mean (SD) or percent; SES socioeconomic status

psychiatric services and healthy controls (3.7 and 4.6\%, respectively).

\section{HRQoL and SDQ}

Table 2 presents means for HRQoL and SDQ, by clinical status and gender. In general, CSHCN who required psychiatric services had the lowest HRQoL scores and the highest SDQ scores among the three groups. In addition, gender differences were observed. Among girls, CSHCN requiring psychiatric services had significantly lower scores in all five domains of HRQoL compared to the control group, whereas male CSHCN requiring psychiatric services had significantly lower HRQoL in only three of the five domains ("psychological well-being," "social support and peers," and "school environment") relative to controls $(P<0.05)$. Female CSHCN without psychiatric service use reported significantly lower HRQoL in the domains of "physical well-being," "autonomy and parent relations," and "school environment" relative to controls $(P<0.05)$, whereas differences in HRQoL between male CSHCN without psychiatric services and controls were not significant.
Similarly, female CSHCN requiring psychiatric services had higher SDQ scores in all domains of the SDQ except the "prosocial subscale," whereas male $\mathrm{CSHCN}$ requiring psychiatric services had higher SDQ scores in just two domains ("emotional problems" and "peer problems") relative to controls.

Female CSHCN not using psychiatric services had higher SDQ scores only in the "hyperactivity" domain and lower SDQ score in "prosocial" domain $(P<0.05)$, whereas their male counterparts had higher SDQ scores only in the "emotional problems" and "prosocial" domains $(P<0.05)$.

The correlations between SDQ and HRQoL are shown in Table 3. In general, correlations within domains measured by both the SDQ and HRQoL were higher than correlations between the various domains within either instrument. The highest correlations between sub-domains were identified between "psychological well-being" in the HRQoL and "emotional problems" in the SDQ $(r=$ -0.57); "social support and peers" in HRQoL and "peer problems" in the SDQ $(r=-0.48)$; and "school environment" in the HRQoL and "hyperactivity" in the SDQ $(r=-0.41)$. 
Table 2 Standardized raw scores for HRQoL and raw scores for the SDQ, by gender and clinical status

\begin{tabular}{|c|c|c|c|c|c|c|c|c|c|c|c|c|}
\hline & \multicolumn{6}{|l|}{ Girls } & \multicolumn{6}{|l|}{ Boys } \\
\hline & \multicolumn{2}{|c|}{$\begin{array}{l}\mathrm{CSHCN} \\
\text { requiring } \\
\text { psychiatric } \\
\text { services }\end{array}$} & \multicolumn{2}{|c|}{$\begin{array}{l}\text { CSHCN } \\
\text { without } \\
\text { psychiatric } \\
\text { services }\end{array}$} & \multicolumn{2}{|c|}{ Controls } & \multicolumn{2}{|c|}{$\begin{array}{l}\mathrm{CSHCN} \\
\text { requiring } \\
\text { psychiatric } \\
\text { services }\end{array}$} & \multicolumn{2}{|c|}{$\begin{array}{l}\mathrm{CSHCN} \\
\text { requiring } \\
\text { psychiatric } \\
\text { services }\end{array}$} & \multicolumn{2}{|c|}{ Controls } \\
\hline & Mean & SD & Mean & SD & Mean & SD & Mean & SD & Mean & SD & Mean & SD \\
\hline \multicolumn{13}{|l|}{ KIDSCREEN-27 } \\
\hline Physical well-being & 61.97 & 22.53 & 69.20 & 16.20 & 77.05 & 15.66 & 75.68 & 17.22 & 75.91 & 14.46 & 78.23 & 15.65 \\
\hline Psychological well-being & 70.74 & 19.14 & 82.20 & 12.07 & 83.63 & 12.90 & 78.86 & 14.99 & 83.48 & 10.18 & 85.80 & 10.30 \\
\hline Autonomy and parent relationship & 74.29 & 17.41 & 80.26 & 13.78 & 84.24 & 12.03 & 80.86 & 13.57 & 82.04 & 13.46 & 82.93 & 11.99 \\
\hline Social support and peers & 75.78 & 19.36 & 83.93 & 13.05 & 84.97 & 13.22 & 75.50 & 20.22 & 81.01 & 14.41 & 82.78 & 15.14 \\
\hline School environment & 73.32 & 20.73 & 73.68 & 16.69 & 80.58 & 14.64 & 72.03 & 17.25 & 78.07 & 14.97 & 77.25 & 14.93 \\
\hline \multicolumn{13}{|l|}{ SDQ } \\
\hline Total difficulties & 14.54 & 5.29 & 10.50 & 4.86 & 9.18 & 4.97 & 12.27 & 5.66 & 10.2 & 4.48 & 9.45 & 4.35 \\
\hline Conduct problems & 2.76 & 2.28 & 1.85 & 1.33 & 1.80 & 1.48 & 2.22 & 1.65 & 1.93 & 1.33 & 1.99 & 1.43 \\
\hline Emotional problems & 4.25 & 2.29 & 2.69 & 2.17 & 2.23 & 2.02 & 2.85 & 2.05 & 2.07 & 1.58 & 1.65 & 1.59 \\
\hline Hyperactivity & 4.49 & 1.51 & 4.24 & 2.28 & 3.40 & 2.06 & 4.48 & 2.15 & 4.18 & 2.12 & 3.90 & 1.99 \\
\hline Peer problems & 3.04 & 1.99 & 1.71 & 1.45 & 1.75 & 1.53 & 2.72 & 2.02 & 2.02 & 1.87 & 1.91 & 1.70 \\
\hline Prosocial behavior & 8.27 & 1.76 & 7.99 & 1.62 & 8.44 & 1.44 & 7.52 & 1.74 & 7.83 & 1.56 & 7.31 & 1.66 \\
\hline
\end{tabular}

$S D Q$ Strength and Difficulties Questionnaire

The results of multiple regression analyses for HRQoL are shown in Table 4. In general, CSHCN utilizing psychiatric services reported significantly lower HRQoL in all five domains, relative to controls. A significant interaction between gender and clinical status was revealed in the domains of "physical well-being" and "autonomy and parental relationships," indicating that the differences in HRQoL between CSHCN requiring psychiatric services and healthy controls were more pronounced among girls than boys. Low family affluence was associated with lower HRQoL in the domains of "physical well-being," "psychological well-being," and "autonomy and parental relationships." Older children reported lower HRQoL in the domains of "physical well-being," psychological well-being," "social support and peers," and "social environment."

\section{Conclusions and discussion}

The present study used a community-based sample to compare children's self-reported HRQoL between CSHCN requiring psychiatric services, $\mathrm{CSHCN}$ not using psychiatric services, and healthy children. Our results are consistent with those of previous studies [16, 26], in which CSHCN have reported lower HRQoL than healthy children. Our study further compared HRQoL between CSHCN who require psychiatric services and those who do not. The results indicate that $\mathrm{CSHCN}$ requiring psychiatric services are especially vulnerable to various difficulties. They reported the lowest HRQoL and most behavioral and emotional problems of our three subject groups. In addition, we discovered significant interaction effects between gender and clinical status that indicated that differences in HRQoL between CSHCN requiring psychiatric services and healthy controls are more pronounced in girls than in boys. Previous studies that have examined gender and age differences in HRQoL have concluded that boys and girls have similar HRQoL at a young age and that HRQoL decreases in adolescence [36-38]. However, the decline in HRQoL with age is more pronounced among girls than boys, especially in psychological and physical dimensions [36]. Our study further found that adolescent girls who require mental health services are especially vulnerable. These findings suggest that gender difference must be taken into consideration in the research and clinical practice of assessing HRQoL among adolescents, especially among those who require mental health services.

Our study also revealed that the most significant correlate of HRQoL is family affluence. These results are consistent with previously published studies $[37,38]$ in which low SES was found to be associated low HRQoL. It is interesting to note that no significant difference in HRQoL existed between those belonging to families classified as of "medium" versus "high" affluence, when affluence was measured using the FAS. For this reason, we grouped these two socioeconomic classes together in our final multiple regression model and found that only children of low 
Table 3 Pearson correlation coefficients for the SDQ and KIDSCREEN-27 (standardized raw scores)

\begin{tabular}{|c|c|c|c|c|c|c|c|c|c|c|c|}
\hline & \multicolumn{6}{|l|}{ SDQ } & \multicolumn{5}{|c|}{ KIDSCREEN-27 } \\
\hline & Total & Conduct & Emotion & Hyper & Peer & Prosoc & Phy & Psy & Par & Soc & Sch \\
\hline \multicolumn{12}{|l|}{ SDQ } \\
\hline \multicolumn{12}{|l|}{ Total } \\
\hline Conduct & $0.67 * *$ & & & & & & & & & & \\
\hline Emotion & $0.74 * *$ & $0.3 * *$ & & & & & & & & & \\
\hline Hyper & $0.69 * *$ & $0.38 * *$ & $0.31 * *$ & & & & & & & & \\
\hline Peer & $0.62 * *$ & $0.26 * *$ & $0.36 * *$ & $0.11 * *$ & & & & & & & \\
\hline Prosoc & $-0.24 * *$ & $-0.31 * *$ & -0.02 & $-0.18 * *$ & $-0.17 * *$ & & & & & & \\
\hline \multicolumn{12}{|c|}{ KIDSCREEN-27 } \\
\hline Phy & $-0.40 * *$ & $-0.22 * *$ & $-0.38 * *$ & $-0.22 * *$ & $-0.28 * *$ & $0.19 * *$ & & & & & \\
\hline Psy & $-0.54 * *$ & $-0.29 * *$ & $-0.57 * *$ & $-0.25 * *$ & $-0.36 * *$ & $0.14 * *$ & $0.5^{* *}$ & & & & \\
\hline Par & $-0.48 * *$ & $-0.36 * *$ & $-0.4 * *$ & $-0.28 * *$ & $-0.26 * *$ & $0.15 * *$ & $0.39 * *$ & $0.54 * *$ & & & \\
\hline Soc & $-0.36 * *$ & $-0.13 * *$ & $-0.28 * *$ & $-0.10^{*}$ & $-0.48 * *$ & $0.2 * *$ & $0.36 * *$ & $0.43 * *$ & $0.34 * *$ & & \\
\hline Sch & $-0.5 * *$ & $-0.36^{* *}$ & $-0.38 * *$ & $-0.41 * *$ & $-0.25 * *$ & $0.26 * *$ & $0.46 * *$ & $0.47 * *$ & $0.45 * *$ & $0.33 * *$ & \\
\hline
\end{tabular}

SDQ Strength and Difficulties Questionnaire; Conduct conduct problems; Emotion emotional problems; Hyper hyperactivity; Peer peer problems; Prosoc prosocial behavior; Phy physical well-being; Psy psychological well-being; Par autonomy and parental relationships; Soc social support and peers; Sch school environment

$* P \leq 0.05 ; * * P \leq 0.01$

Table 4 Multiple regression for health-related quality of life

\begin{tabular}{|c|c|c|c|c|c|c|c|c|c|c|c|c|c|c|c|}
\hline \multirow[t]{2}{*}{ Variable } & \multicolumn{3}{|c|}{ Physical well-being } & \multicolumn{3}{|c|}{$\begin{array}{l}\text { Psychological } \\
\text { well-being }\end{array}$} & \multicolumn{3}{|c|}{$\begin{array}{l}\text { Autonomy and parent } \\
\text { relationship }\end{array}$} & \multicolumn{3}{|c|}{ Social support and peers } & \multicolumn{3}{|c|}{ School environment } \\
\hline & $\beta$ & SE & $P$ value & $\beta$ & SE & $P$ value & $\beta$ & SE & $P$ value & $\beta$ & SE & $P$ value & $\beta$ & SE & $P$ value \\
\hline Intercept & 107.17 & 6.03 & $<0.0001$ & 97.5 & 4.67 & $<0.0001$ & 83.77 & 0.91 & $<0.0001$ & 93.04 & 5.75 & $<0.0001$ & 107.1 & 5.84 & $<0.0001$ \\
\hline $\begin{array}{l}\text { CSHCN with } \\
\text { psychiatric } \\
\text { services }\end{array}$ & -2.84 & 2.29 & 0.2155 & -6.59 & 1.77 & 0.0002 & -2.44 & 1.87 & 0.1927 & -7.66 & 1.82 & $<0.0001$ & -6.16 & 1.83 & 0.0008 \\
\hline $\begin{array}{l}\text { CSHCN without } \\
\text { psychiatric } \\
\text { services }\end{array}$ & -4.49 & 1.5 & 0.0029 & -1.91 & 1.16 & 0.1011 & -2.04 & 1.23 & 0.0966 & -1.46 & 1.43 & 0.3092 & -2.35 & 1.44 & 0.1025 \\
\hline Girl & -2.83 & 1.38 & 0.0415 & -2.07 & 1.07 & 0.0539 & 0.23 & 1.13 & 0.8409 & 2.2 & 1.24 & 0.0761 & & & \\
\hline $\begin{array}{l}\text { CSHCN with } \\
\text { psychiatric } \\
\text { services* girl }\end{array}$ & -10.33 & 3.93 & 0.0088 & -5.8 & 3.04 & 0.0566 & -6.81 & 3.23 & 0.0357 & & & & & & \\
\hline Age in years & -2.14 & 0.46 & $<0.0001$ & -0.89 & 0.36 & 0.0124 & & & & -0.79 & 0.44 & 0.0709 & -2.17 & 0.45 & $<0.0001$ \\
\hline $\begin{array}{l}\text { Low family } \\
\text { affluence } \\
\text { (FAS; low vs. } \\
\text { medium/high) }\end{array}$ & -7.87 & 2.95 & 0.0079 & -4.65 & 2.32 & 0.0458 & -5.77 & 2.43 & 0.0181 & & & & & & \\
\hline
\end{tabular}

family affluence reported a significantly lower HRQoL. In addition, more families from $\mathrm{CSHCN}$ requiring psychiatric services were classified as families of "low affluence" compared to $\mathrm{CHSCN}$ not using psychiatric services and healthy children. Among those $\mathrm{CSHCN}$ requiring psychiatric services, $8.0 \%$ were considered poor, versus only $3.7 \%$ of CSHCN not using psychiatric services and $4.6 \%$ of healthy children.
It is important to note that $75 \%$ of our study participants were classified in the category "high family affluence." Our study sample is drawn from the Canton of Zurich, which included a majority of urban and well-developed communities. This could explain why such a high percentage of our participants were classified as living in a highly affluent family. The distribution of study participants classified as "high," "medium," and "low" family 
affluence in our study is 75,20 , and $5 \%$, respectively. This contrasts with the results of another national survey that involved a representative national sample in Switzerlandthe 2005/2006 Health Behavior in School-Age Children (HBSC) Survey, a World Health Organization sponsored international survey across more than 40 countries, which drew a national sample of school-age children 11-15 years old. The distribution of FAS in the participants of the HBSC Survey was 48, 43, and 9\%, respectively. Although families of low affluence could be slightly underrepresented in our sample due to the issues of non-response and urban sampling, our results are consistent with previous studies that have demonstrated an association between low family affluence and lower HRQoL [37, 38]. However, families of medium affluence in our study were underrepresented compared to the national sample (20\% vs. $43 \%)$. This may explain why we failed to find any significant difference in HRQoL between families of high and medium affluence, which is contrary to previous studies that identified a gradient relationship of family affluence with HRQoL [37-39] and with multiple health complaints among adolescents [40].

In conclusion, using a community-based sample (instead of a clinical sample, as typically utilized in previous studies), we found that CSHCN who use psychiatric services report a much lower level of HRQoL and more emotional and behavioral problems than either CSHCN who do not use psychiatric services or healthy controls. They are, hence, the most vulnerable group. In particular, a greater number of less affluent families are in this versus the other two groups. However, there is a general lack of research focusing specifically on HRQoL in such children $[13,14]$. More research on HRQoL in this population of children could help to improve therapies and advance our knowledge of the reasons for and consequences of mental illness among them [11].

Our results should be interpreted with caution, however. Due to our study design, the CSHCN we studied who required psychiatric services could represent a mixed sample. We are not able to determine whether the needs for treatment and/or counseling for psychological problems were the consequences of other physical chronic conditions. In addition, because of the nature of cross-sectional studies, we also could not determine whether the needs for treatment and/or counseling for psychological problems were the consequences of material deprivation due to low family SES or themselves contributing to low family affluence. Additionally, our sample of $\mathrm{CSHCN}$ requiring psychiatric services consisted of only 88 children, and only 26 girls. Consequently, more studies are needed to investigate gender effects. Furthermore, we cannot rule out the possibility that the $\mathrm{CSHCN}$ requiring psychiatric services had a lower HRQoL relative to our other two subject groups because of a relatively high conceptual relationship between mental disorders and HRQoL [21]. However, other authors [19] have identified lower HRQoL even after controlling for such factors. Therefore, it is plausible that the differences we found did not occur solely because of such conceptual overlapping.

Finally, despite the above-mentioned limitations, we feel that the results of our study still may be generalized to other developed countries like those of Scandinavia and elsewhere in Western Europe, countries with similar distributions of family affluence. Children and especially girls with extreme material deprivation in such well-developed countries could be especially vulnerable to mental health problems, relative to their counterparts. Therefore, gender difference and family affluence should be taken into consideration in clinical settings, including any intervention program. It is possible that we failed to identify the expected gradient relationship between family affluence and HRQoL among CSHCN requiring psychiatric services because families classified as medium family affluence were underrepresented in our sample. Further research with a more representative, national sample would provide a better opportunity to uncover any such gradient effect. In addition, further research adopting a longitudinal design would help to better understand any causal relationships and long-term effects among the most vulnerable group of $\mathrm{CSHCN}$ requiring psychiatric services.

Acknowledgments The study was partially supported by the Wyeth Foundation for the Health of Children.

\section{References}

1. WHO (1946). Constitution, World Health Organization.

2. Frey, M. (1996). Behavioral correlates of health and illness in youths with chronic illness. Applied Nursing Research, 9(4), $167-176$

3. Geist, R., Grdisa, V., \& Otley, A. (2003). Psychosocial issues in the child with chronic conditions. Best Practice \& Research in Clinical Gastroenterology, 17(2), 141-152.

4. Haarasilta, L., Marttunen, M., Kaprio, J., \& Aro, H. (2005). Major depressive episode and physical health in adolescents and young adults: Results from a population-based interview survey. European Journal of Public Health, 15(5), 489-493.

5. Haarasilta, L. M., Marttunen, M. J., Kaprio, J. A., \& Aro, H. M. (2004). Correlates of depression in a representative nationwide sample of adolescents (15-19 years) and young adults (20-24 years). European Journal of Public Health, 14(3), 280-285.

6. Newacheck, P. W., \& Halfon, N. (1998). Prevalence and impact of disabling chronic conditions in childhood. American Journal of Public Health, 88(4), 610-617.

7. Sawyer, S. M., Drew, S., Yeo, M. S., \& Britto, M. T. (2007). Adolescents with a chronic condition: Challenges living, challenges treating. Lancet, 369(9571), 1481-1489.

8. Yeo, M., \& Sawyer, S. (2005). Chronic illness and disability. BMJ, 330(7493), 721-723. 
9. Taylor, R. M., Gibson, F., \& Franck, L. S. (2008). A concept analysis of health-related quality of life in young people with chronic illness. J Clin Nurs, 17(14), 1823-1833.

10. Committee on Psychosocial Aspects of Child, Family Health. (2001). The new morbidity revisited: A renewed commitment to the psychosocial aspects of pediatric care. Pediatrics, 108(5), $1227-1230$

11. Coghill, D., Danckaerts, M., Sonuga-Barke, E., \& Sergeant, J. (2009). Practitioner review: Quality of life in child mental healthconceptual challenges and practical choices. Journal of Child Psychology and Psychiatry, 50(5), 544-561.

12. Schmeck, K., \& Poustka, F. (2006). Quality of life and childhood disorders. In H. Katschnig, H. Freeman, \& N. Sartorius (Eds.), Quality of life in mental disorders (2nd ed.). Chichester, NY: Wiley.

13. Bullinger, M., \& Ravens-Sieberer, U. (1995). General principles, methods and areas of application of quality of life research in children. Praxis der Kinderpsychologie und Kinderpsychiatrie, 44, 391-399.

14. Mattejat, F., Jungmann, J., Meusers, M., Moik, C., Nölkel, P., Schaff, C., et al. (1998). Das Inventar zur Erfassung der Lebensqualität bei Kindern und Jugendlichen (ILK)-Eine Pilotstudie. Eine Zeitschrift für Kinder und Jugendpsychiatrie und Psychotherapie, 26(3), 148-182.

15. Bastiaansen, D., Koot, H. M., \& Ferdinand, R. F. (2005). Psychopathology in children: Improvement of quality of life without psychiatric symptom reduction? European Child and Adolescent Psychiatry, 14(7), 364-370.

16. Bisegger, C., Bernhard, C., \& Kidscreengruppe, E. (2005). Kidscreen: Fragebogen zur Erfassung der gesundheitsbezogenen Lebensqualität von Kindern und Jugendlichen. Manual der deutschsprachigen Version für die Schweiz. Bern: Abteilung für Gesundheitsforschung des Instituts für Sozial- und Präventivmedizin der Universität Bern.

17. Centers for Disease Control and Prevention. (2005). Mental health in the United States: Health care and well being of children with chronic emotional, behavioral, or developmental problemsUnited States, 2001. MMWR Morbidity \& Mortality Weekly Report, 54(39), 985-989.

18. Clark, E. E., Carlisle, S. S., Giard, A., \& Turner, W. M. (2007). Speaking your mind: Measuring the subjective quality of life of children with mental illnesses. Issues Ment Health Nurs, 28(12), 1277-1291.

19. Sawyer, M. G., Whaites, L., Rey, J. M., Hazell, P. L., Graetz, B. W., \& Baghurst, P. (2002). Health-related quality of life of children and adolescents with mental disorders. Journal of the American Academy of Child and Adolescent Psychiatry, 41(5), 530-537.

20. Waters, E., Davis, E., Nicolas, C., Wake, M., \& Lo, S. K. (2008). The impact of childhood conditions and concurrent morbidities on child health and well-being. Child: Care, Health and Development, 34(4), 418-429.

21. Katschnig, H. (2006). How useful is the concept of quality of life in psychiatry? In H. Katschnig, H. Freeman, \& N. Sartorius (Eds.), Quality of life in mental disorders. Chichester: Wiley.

22. Eiser, C., \& Morse, R. (2001). Can parents rate their child's health-related quality of life? Results of a systematic review. Quality of Life Research, 10(4), 347-357.

23. McPherson, M., Arango, P., Fox, H., Lauver, C., McManus, M., Newacheck, P. W., Perrin, J. M., Shonkoff, J. P., \& Strickland, B. (1998). A new definition of children with special health care needs. Pediatrics, 102(1), 137-140.

24. Perrin, E. C., Newacheck, P., Pless, I. B., Drotar, D., Gortmaker, S. L., Leventhal, J., Perrin, J. M., Stein, R. E. K., Walker, D. K., \& Weitzman, M. (1993). Issues involved in the definition and classification of chronic health conditions. Pediatrics, 91(4), 787-793.
25. Davidoff, A. J. (2004). Identifying children with special health care needs in the national health interview survey: A new resource for policy analysis. Health Services Research, 39(1), 53-71.

26. KIDSCREEN Group (2006). The Kidscreen Questionnaires. Quality of life questionnaires for children and adolescents. Lengerich: Pabst Science Publishers.

27. Jann, B. (2007). Überlegungen zum Berner Stichprobenplan. Swiss Journal of Sociology, 33, 307-325.

28. Mohler-Kuo, M., Jann, B., Dey, M., \& Zellweger, U. (2011). A recruitment method to obtain community samples of children for survey research in Switzerland. International Journal of Public Health, 56(3), 353-356.

29. Bethell, C. D., Read, D., Stein, R. E. K., Blumberg, S. J., Wells, N., \& Newacheck, P. W. (2002). Identifying children with special health care needs: Development and evaluation of a short screening instrument. Ambulatory Pediatrics, 2(1), 38-48.

30. Schmidt, S., Thyen, U., Petersen, C., \& Bullinger, M. (2004). The performance of the screener to identify children with special health care needs in a European sample of children with chronic conditions. European Journal of Pediatrics, 163(9), 517-523.

31. Goodman, R. (1997). The strengths and difficulties questionnaire: A research note. Journal of Child Psychology and Psychiatry and Allied Disciplines, 38(5), 581-586.

32. Lai, K. Y. C., Luk, E. S. L., Leung, P. W. L., Wong, A. S. Y., Law, L., \& Ho, K. R. (2010). Validation of the Chinese version of the strengths and difficulties questionnaire in Hong Kong. Social Psychiatry and Psychiatric Epidemiology, 45(12), 1179-1186.

33. Goodman, R., Meltzer, H., \& Bailey, V. (2003). The Strengths and Difficulties Questionnaire: A pilot study on the validity of the self-report version (Reprinted from European Child and Adolescent Psychiatry, 7, 125-130, 1998). International Review of Psychiatry, 15(1-2), 173-177.

34. Currie, C. E., Elton, R. A., Todd, J., \& Platt, S. (1997). Indicators of socioeconomic status for adolescents: The WHO Health Behaviour in School-aged Children Survey. Health Education Research, 12(3), 385-397.

35. Currie, C., Molcho, M., Boyce, W., Holstein, B., Torsheim, T., \& Richter, M. (2008). Researching health inequalities in adolescents: The development of the Health Behaviour in School-Aged Children (HBSC) Family Affluence Scale. Social Science and Medicine, 66(6), 1429-1436.

36. Bisegger, C., Cloetta, B., von Bisegger, U., Abel, T., \& RavensSieberer, U. (2005). Health-related quality of life: Gender differences in childhood and adolescence. Sozial- und Präventivmedizin/Social and Preventive Medicine, 50(5), 281-291.

37. Erhart, M., Ottova, V., Gaspar, T., Jericek, H., Schnohr, C., Alikasifoglu, M., et al. (2009). Measuring mental health and wellbeing of school-children in 15 European countries using the KIDSCREEN-10 Index. International Journal of Public Health, $54,160-166$.

38. van Rueden, U., Gosch, A., Rajmil, L., Bisegger, C., RavensSieberer, U., \& European Kidscreen Group (2006). Socioeconomic determinants of health related quality of life in childhood and adolescence: Results from a European study. Journal of Epidemiology and Community Health, 60, 130-165.

39. Currie, C., Gabhainn, S. N., Godeau, E., Roberts, C., Smith, R., Currie, D., et al. (Eds.) (2008). Inequalities in young people's health: HBSC international report from the 2005/06 survey. Health Policy for Children and Adolescents, No. 5. Copenhagen, Denmark: WHO Regional Office for Europe.

40. Holstein, B., Currie, C., Boyce, W., Damsgaard, M., Gobina, I., Kökönyei, G., et al. (2009). Socio-economic inequality in multiple health complaints among adolescents: International comparative study in 37 countries. International Journal of Public Health, 54, 260-270. 